\title{
Nanoindentation as a Tool for Recognition of Composites Components
}

\author{
M. NowICKI* AND B. Susea \\ Institute of Physics, Poznan University of Technology \\ Nieszawska 13A, 60-965 Poznań, Poland
}

\begin{abstract}
A brief description of the basis principles of determination Young's indentation modulus and hardness by using of nanoindentation technique are given. The detailed indentation technique and characteristic features of polymer at room temperature are also discussed.
\end{abstract}

PACS numbers: 81.05.Lg

\section{Introduction}

Scanning force microscopy (SFM), developed by G. Binnig et al. [1, 2], permits to image surface of a broad scale of materials with nanometer resolution. This technique is also suitable for surface characterization of organic materials [3, 4]. Contrary to other methods such as scanning tunneling microscopy (STM) and transmission electron microscopy (TEM), it does not require any special preparation of the sample. One of the latest extensions of this technique is the nanoindenting SFM (NI-SFM) [5-8]. The HYSITRON-System, the first commercial representative of nanomechanical test equipment of this type, takes advantage of an electrostatic transducer to obtain the topography of the sample surface by SFM in contact mode. Moreover, it is possible to investigate the local mechanical properties of samples [9] at any selected point. This technique has been successfully used for the study of various nanomechanical properties of polymer single crystals and amorphous polymer thin films, including elasticity [10, 11], hardness [12] and viscoelasticity measurements $[13,14]$. Depth sensing nanoindentation differs from classical hardness measurements (Vickers, Brinell and Knoop) [15], where the impressions are first generated, and then imaged using a microscopy technique. Load and penetration depths are simultaneously recorded during both loading and unloading, resulting in a force-depth-diagram. This diagram provides much more

*corresponding author; e-mail: mnowicki@phys.put.poznan.pl 
information than a microscopy image of the impression since it tells us the "story" of the elastic and plastic deformation with increasing load (Fig. 1) and permits the determination of hardness and Young's indentation modulus as a function of penetration depth. Moreover, the hardness measurements can be made with penetrations smaller than $1 \mu \mathrm{m}$.

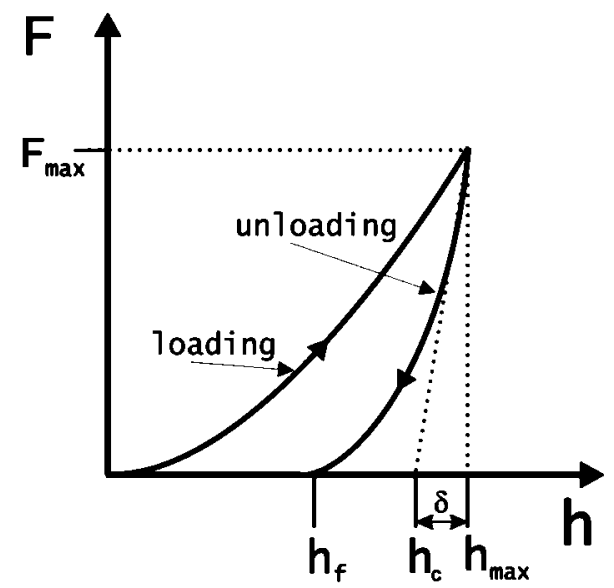

Fig. 1. Schematic representation of the force-depth curve for a nanoindentation procedure. The symbol $\delta$ representing an elastic deformation part of the indent.

In this paper, the principle of the determination Young's indentation modulus and hardness will be briefly explained. An atomic force microscopy measurements and characteristic features (Young's indentation modulus, hardness) of the polymer surface at room temperature are also discussed.

\section{Experimental part}

\subsection{Experimental equipment}

All experiments were performed using a Hysitron TriboScope (electrostatic transducer) attached to a scanning force microscope with a controller Nanoscope $\mathbf{E}$ of the Veeco Metrology Group. The heart of the testing instruments is a three-plate capacitive force/displacement transducer. The three-plate capacitive design provides a high sensitivity, a large dynamic range, and a linear force or displacement output signal. The sensor consists of two fixed outer electrodes (drive plates) which are driven by $\mathrm{AC}$ signals $180^{\circ}$ out of phase with each other. Since the drive plates are parallel to each other and closely spaced with respect to the lateral dimensions, the electric field potential between the plates varies linearly. Since the signals applied to the drive plates are equal in magnitude but opposite in polarity at any instant, the electric field potential is maximized (equal to the applied signal) at the drive plates and minimized (zero, the two opposite polarity signals cancel each 
other out) at the site centered directly between the drive plates. That allows us to get "Z" information. Connecting high DC voltage to driving plates a user can apply a defined force to the tip. This force is proportional to the squared DC voltage. The tip is made of diamond having a shape of a cube corner (three-sided pyramid). They were carried out at room temperature in air atmosphere. The following parameters were obtained (Fig. 1): maximum indentation depth $\left(h_{\max }\right)$, contact depth $\left(h_{\mathrm{c}}\right)$, depth of the remaining impression after complete unloading $\left(h_{f}\right)$, maximal applied force $\left(F_{\max }\right)$, nano-hardness and Young's indentation modulus.

The key quantities to determine the mechanical properties are defined as follows. The maximum indentation depth $h_{\max }$ includes elastic and plastic deformation. The depth at which the applied forces becomes zero on unloading is called $h_{\mathrm{f}}$. The depth $h_{\mathrm{c}}$ is the contact depth at which the cross-section area $A_{\mathrm{c}}$ is taken to calculate hardness and indentation modulus. The nano-hardness of the sample $\left(H_{\mathrm{N}}\right)$ is determined using the following formula:

$$
H_{\mathrm{N}}=\frac{F_{\max }}{A_{c}\left(h_{c}\right)}
$$

where $F_{\max }$ is the maximum applied load and $A_{c}$ is the cross-sectional area corresponding to the depth $h_{\mathrm{c}}$ (see Fig. 1). The determination of the contact depth $h_{\mathrm{c}}$ is given by $[16]$ :

$$
h_{\mathrm{c}}=h_{\max }-0.75 \frac{F_{\max }}{S},
$$

where $S$ is the contact stiffness,

$$
S=\frac{\mathrm{d} F}{\mathrm{~d} h}
$$

with $\mathrm{d} F / \mathrm{d} h$ being the slope of the unloading curve at the initial point of unloading. The reduced Young's indentation modulus $E_{\mathrm{r}}$ is a measure of the elastic properties of the tip-sample system and can be calculated from the load-depth curves according to the following formula:

$$
E_{\mathrm{r}}=\frac{1}{2} \sqrt{\frac{\pi}{A_{\mathrm{c}}\left(h_{\mathrm{c}}\right)}} \frac{\mathrm{d} F}{\mathrm{~d} h} .
$$

For elastically deformable indenters, the reduced modulus $E_{\mathrm{r}}$ can be generalized and is defined as

$$
\frac{1}{E_{\mathrm{r}}}=\frac{1-\nu_{\mathrm{s}}^{2}}{E_{\mathrm{s}}}+\frac{1-\nu_{\mathrm{t}}^{2}}{E_{\mathrm{t}}}
$$

where $E_{\mathrm{s}}$ and $\nu_{\mathrm{s}}$ are Young's indentation modulus and Poisson ratio of the sample, $E_{\mathrm{t}}$ and $\nu_{\mathrm{t}}$ are Young's indentation modulus and Poisson ratio of the indenter tip. Since $E_{\mathrm{t}}$ is much higher than $E_{\mathrm{s}}$ the value of $E_{\mathrm{r}}$ will hardly differ from $E_{\mathrm{s}}$. 


\subsection{Calibration of the nanoindenter}

For a tip of ideal cube corner pyramid geometry the relation between indentation depth $(h)$ and cross-section area (tip area function $A_{\mathrm{C}}$ ) is

$$
A_{\mathrm{c}}=2.598 h^{2} \text {. }
$$

The tip does not have an ideal shape but is blunted due to micromechanical generation of the tip shape and the use for imprinting into a material. A typical value of the blunting radius of the tip is $100 \mathrm{~nm}$ and, though the tip is made of diamond, the radius of curvature will increase during a frequent use. Thus, the determination of the real relation $A_{c}\left(h_{c}\right)$ is necessary for proper calculation of nano-hardness and Young's indentation modulus, particular in case of small penetrations.

To calibrate the tip, a series of indentations of different loads and depths into a specimen of known mechanical properties was performed. Then the area $A_{c}$ as a function of depth can be determined such that the correct values of the sample are obtained. Since hardness is a quantity that depends on real structure of the material and is normally subject to an indentation size effect, this entity is not suited for calibration. Better suited is the elastic modulus which is hardly influenced by nanoscopic defects and should be constant. The test specimen should be homogeneous and isotropic, and should not be subject to chemical alterations, i.e. oxidation. A suited material is fused quartz, which also gives a large elastic redeformation on unloading (very ductile materials with a small contribution of

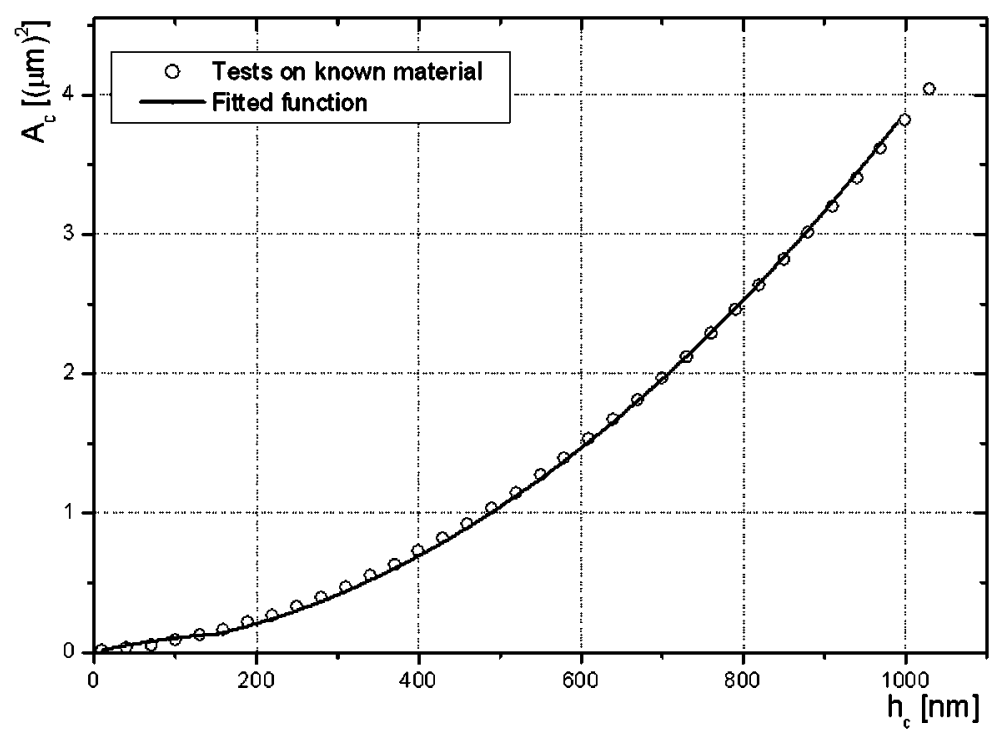

Fig. 2. Area function determined by indentation of fused quartz. 
the elastic deformation to the total penetration, as copper, e.g., are not suited for this purpose). A series of 50 tests was made on fused quartz with the following parameters:

Young's modulus $E_{0}=72 \mathrm{GPa}$, Poisson's number $\nu_{0}=0.17$.

This results in 50 data pairs $\left(h_{i}, A_{\mathrm{c}}\left(h_{i}\right)\right), i=1, \ldots 50$. The data can then be fitted to any suitable function. This function should include a parabolic term (representing the ideal pyramid), a linear term (representing a spherical blunting) and other terms characterizing tip truncation. We used a following expression [16]:

$$
A_{c}=a_{0} h^{2}+a_{1} h+a_{2} h^{1 / 2}+a_{3} h^{1 / 4}+a_{4} h^{1 / 8}+a_{5} h^{1 / 16}
$$

and determined following coefficients:

$$
A_{\mathrm{c}}=3.37 h^{2}+4.12 \times 10^{2} h-4.88 \times 10^{2} h^{1 / 2}+1.78 \times 10^{3} h^{1 / 16} .
$$

The calculated area function for our tip is shown in Fig. 2.

\section{Results}

\subsection{Detection of $\mathrm{SiO}_{2}$ particles in polyethylene terephthalate matrix}

The silicon dioxide is added to polymers as a filling material very often. The aim of this process is changing mechanical properties or reduction of production coast. Homogeneity arrangement of $\mathrm{SiO}_{2}$ particles in polymer matrix is also very important. Generally, atomic force microscopy allows us to investigate arrangement of particles added to composite, but the observation of topography only could lead to some errors during analysis of filling material distribution in polymer.

The application of nanoindentation technique improves identification which surface structure is built from which material. Mostly, on the measured sample hardness and Young's modulus for spherical structures are the same as for $\mathrm{SiO}_{2}$. Figure 3a shows topography (2D image) of polystyrene sample with $\mathrm{SiO}_{2}$ particles in a shape of spheres of diameter from 0.5 to $3 \mu \mathrm{m}$. On the ground of topography image we could suggest that we can see one sphere on the polymer surface. However, nanoindentation test on the surface of sphere (Fig. 3b) and test on the surrounding surface (Fig. 3c) give us identical mechanical parameters which are typical of polystyrene. It was nano-hardness of about $0.15 \mathrm{GPa}$ and Young's modulus of $2.3 \mathrm{GPa}$. Hence, nanoindentation technique allows getting more precisely information about the observed structures of materials in nanoscale.

\subsection{Recognition of components in polymer composite}

Recognition of components on a sample built of two materials with similar mechanical properties is much more complicated. A mixture of two polymers can be an example of such material. In this instance when mechanical properties are almost the same, it can turn out that we need to make tests also on pure (non 

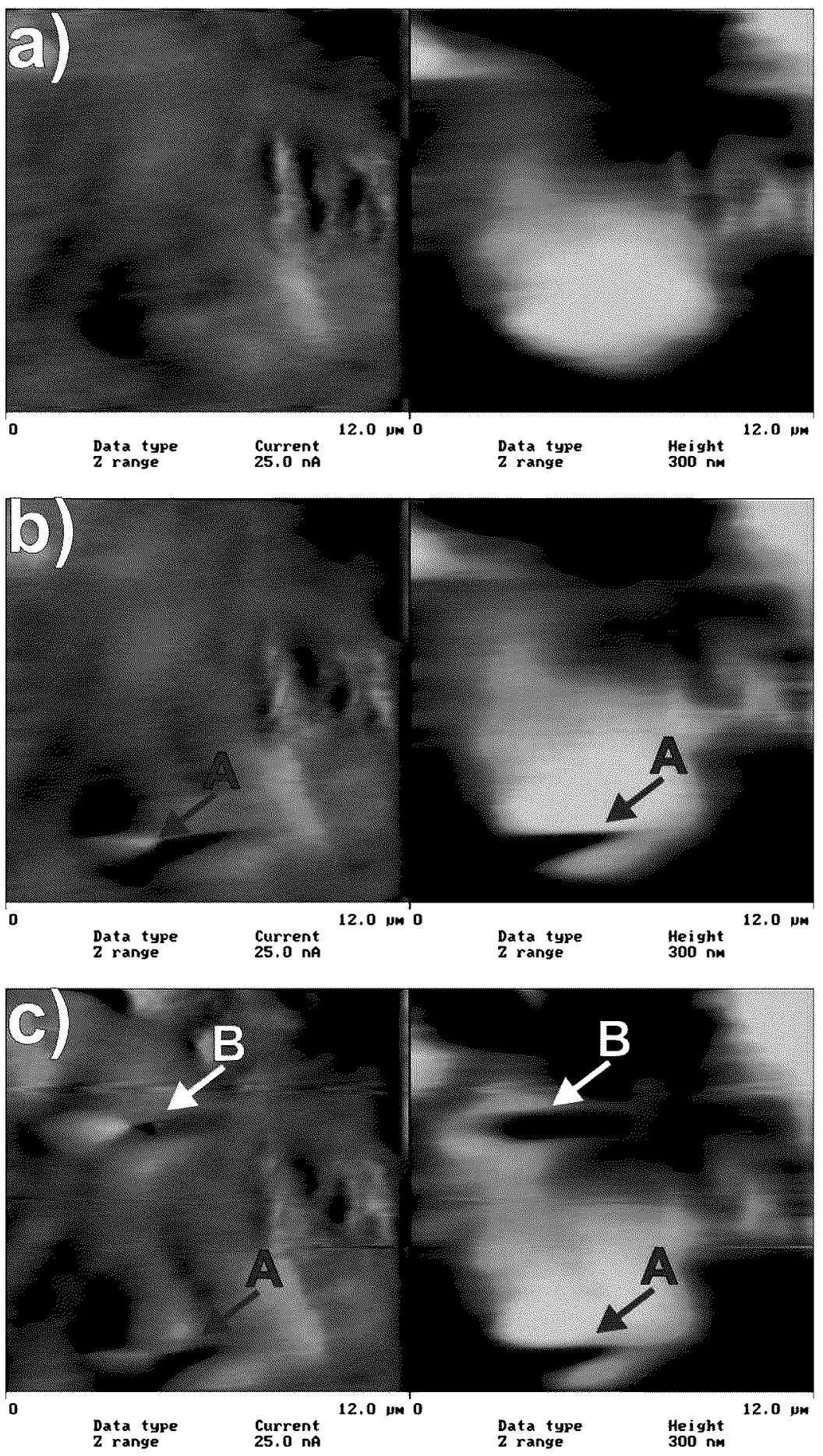

Fig. 3. Detection of $\mathrm{SiO}_{2}$ spheres in polystyrene matrix. 

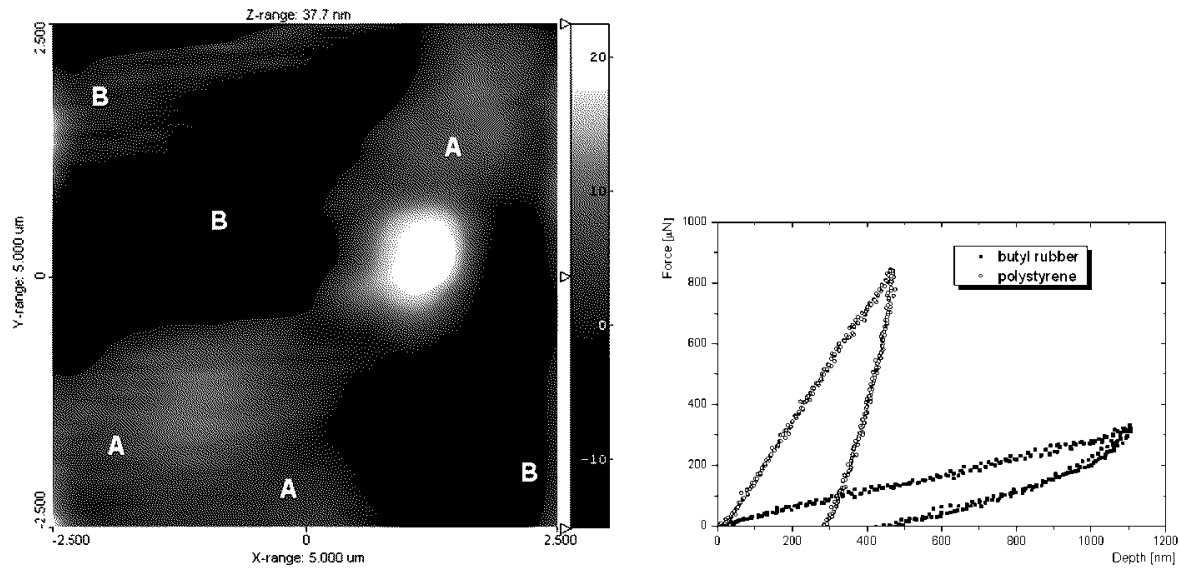

Fig. 4. Detection of polymers distribution in polystyrene/butyl rubber mixture. Places marked as $A$ contains polystyrene, $B$ - rubber. On the diagram are shown typical force-depth curves for both materials.

mixed) materials. It allows us to identify structures not only from mechanical properties, but also from the shape of force-depth curve. If differences between measured materials are more significant we can simply identify structures by determining their mechanical properties. Figure 4 shows an image of the surface polystyrene/butyl rubber mixture. Spots marked as $A$ are built from polystyrene, $B$ indicated butyl rubber. There are points of real measurement. The measured values of Young's indentation modulus for polystyrene are $2.22 \mathrm{GPa}$ and $0.1 \mathrm{GPa}$ for butyl rubber, nano-hardness $-0.15 \mathrm{GPa}$ and $0.01 \mathrm{GPa}$, respectively. These values are the same as the data from the literature $[17,18]$. In this way, we can get a map of the mechanical properties for investigated sample which shows distribution of components on a sample surface.

\section{Summary}

The application of nanoindentation technique allows us to define precisely mechanical properties of polymers. Local measurements enable recognition, which kind of material is built on a surface of the sample. Thanks to nanoindentation technique we receive more information about the structure of the sample than we can get using atomic force microscopy.

\section{Acknowledgment}

A part of the work has been performed with support from the Poznan University of Technology under the project PB 62-198/03-BW. 


\section{References}

[1] G. Binnig, H. Rohrer, Helv. Phys. Acta 55, 726 (1982).

[2] G. Binnig, C.F. Quate, C. Gerber, Phys. Rev. Lett. 56, 933 (1986).

[3] S.N. Magonov, D.H. Reneker, Annu. Rev. Mater. Sci. 27, 175 (1997).

[4] M.C. Goh, in: Advance in Chemical Physics, Eds. I. Prigogine, S.A. Rice, Vol. 91, John Wiley \& Sons, 1995, p. 1.

[5] J. Mater. Res. 14, (1999), the whole volume.

[6] B. Wolf, A. Richter, New J. Phys, 5, 15.1 (2003).

[7] A. Richter, B. Wolf, R. Smith, in: Proc. MRS Spring Meeting 2003, Eds. K.J. Hemker, D.H. Lassila, L.E. Levine, H.M. Zbib, Vol. 779, MRS, Warrendale (USA) 2003, p. 5.14.

[8] P. Maier, A. Richter, R.G. Faulkner, R. Ries, Mater. Charact. 48, 329 (2002).

[9] B. Capella G. Dietler, Surf. Sci. Rep. 34, 1 (1999).

[10] B. Du, J. Liu, Q. Zhang, T. He, Polymer 42, 5901 (2001).

[11] B.J. Du, J. Zhang, Q. Zhang, D. Yan, T. He, O.K. Tsui, Macromol. 33, 7521 (2000).

[12] D. Drechsler, A. Karbach, H. Fuchs, Appl. Phys. A 66, 825 (1998).

[13] T. Kajiyama, K. Tanaka, A. Takahara, Macromol. 31, 5150 (1998).

[14] O.K.C. Tsui, X.P. Wang, J.Y.L. Ho, T.K. Ng, X.D. Xiao, Macromol. 33, 4198 (2000).

[15] The UK's National Measurement Laboratory http://www.npl.co.uk/force/ guidance/hardness/.

[16] W.C. Oliver, G.M. Pharr, J. Mater. Res. 7, 1564 (1992).

[17] GoodFellow Catalogue, www.gooodfellow.com.

[18] USA Plastics, www.plasticsusa.com. 